\title{
Análisis morfométrico de la microcuenca "C" núcleo Cunha, São Paulo, Brasil
}

\author{
Cerignoni, F.J. ${ }^{* 1}$, Rodrigues, V.A. ${ }^{2}$ \\ ${ }^{1}$ Estudiante $5^{o}$ añoIngenieríaForestal - Depto Ciência Forestal - Faculdade de Ciências Agronômicas, UNESP - \\ Botucatu - São Paulo - Brasil. \\ 2 2Ingeniero Forestal - Profesor Adjunto - Depto Ciência Forestal - Faculdade de Ciências Agronômicas, \\ UNESP - Botucatu - São Paulo - Brasil. \\ *e-mail: fcerignoni@gmail.com
}

\section{Resumen}

El presente trabajo tiene como objetivo analizar la morfometría de la microcuenca con ayuda del Sistema de Información Geográfica (SIG), para estimar los parámetros dimensionales, la densidad de drenaje, así como analizar las características de la pendiente, a través de la caracterización de las dimensiones de datos altimétricos y coeficiente de rugosidad. La microcuenca "C" se encuentra en el "Parque Estadual da Serra do Mar - Núcleo / Cunha", en el municipio del Cunha, São Paulo, Brasil. Los parámetros morfométricos fueron obtenidos del mapa topográfico editado por el Instituto Geográfico y Cartográfico (IGC), aescala 1:10.000, disponible en el sitio web Geoportaly, georreferenciado utilizando el software ArcGIS 10.1. Los resultados del alto coeficiente de rugosidad $(\mathrm{Rn}=101.88)$ encuadran la microcuenca en la clase $\mathrm{D}$, con suelo favorable para la reforestación. Los resultados del factor de forma $(\mathrm{Ff}=0.66)$, índice de circularidad $(\mathrm{Ic}=0.60)$, coeficiente de compacidad $(\mathrm{kc}=1.30)$ y la densidad de drenaje $(\mathrm{Dd}=1.8) \mathrm{km} \mathrm{km}^{-2}$, indican una tendencia de inundaciones mediana. Por lo tanto, la microcuenca hidrográfica debe permanecer con la protección del suelo en el relieve montañoso con bosques Atlánticos, pues, con la alta pendiente de 57.7\%, la cuenca es susceptible a la escorrentía del agua, erosión y sedimentación de los ríos, durante las altas precipitaciones.

Palabras clave: bosque atlántico,geoprocesamiento, microcuenca, morfometría, SIG. 


\section{Introducción}

El aumento de la demanda de agua, debido al crecimiento acelerado de la población en el mundo ha provocado, en muchas regiones, la escasez de recursos hídricos. El agua es la materia prima fundamental para la vida, siendo un elemento irremplazable en las diversas actividades humanas, además de mantener el equilibrio del

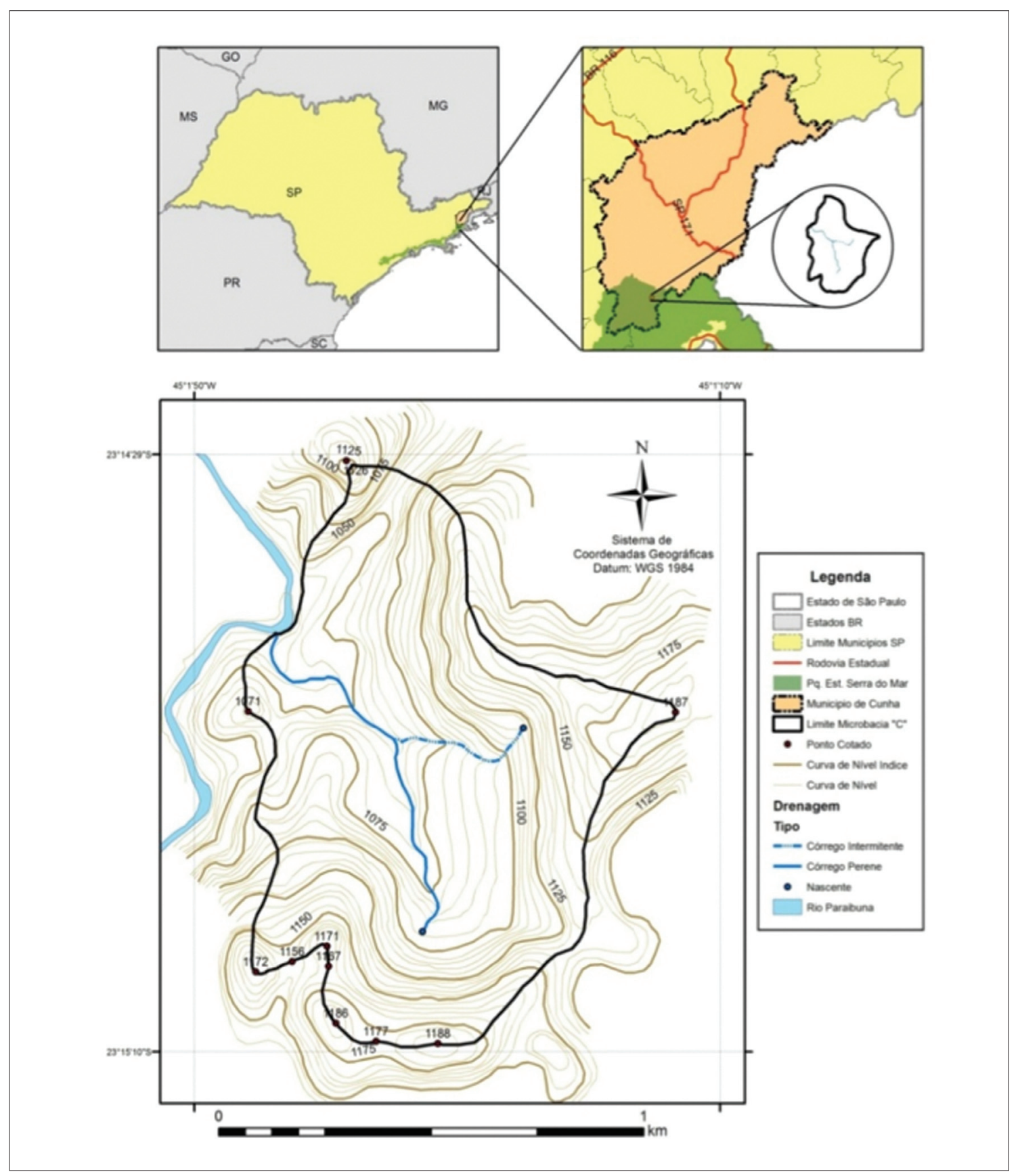

Figura 1. Mapa de localización de la cuenca "C", límite topográfico, contornos, red de drenaje y puntos de elevación. 
medio ambiente. El deterioro de los recursos naturales, especialmente los recursos hídricos es una de las mayores preocupaciones de hoy (Carvalho et al., 2010).

Según Feitosa et al., (2011), la dinámica ambiental de un área puede ser estudiada mediante la caracterización de su paisaje y elementos, e incluso puede ser analizada a través de serie de parámetros. Un tipo de análisis posible es el análisis morfométrico, cuyo objetivo general es el análisis de la disposición y distribución de ciertos elementos en el paisaje. Para ello, se utilizan índices que relacionan estos elementos del paisaje con sus atributos (Cherem et al., 2011).

Una cuenca se puede definir como una cierta área drenada por un río, o una red de vías constituida por un conjunto de canales de flujo interconectados y confinados dentro de un área dada limitada por la topografía. Según Cherem et al., (2011), una de las unidades espaciales adoptadas en el análisis morfométrico es la divisoria de aguas. Ello facilita el estudio de las relaciones de causa-efecto entre las de cabecera y desembocadura de la red de drenaje.

De acuerdo con Rodrigues (2004, 2013), los estudios en cuencas comienzan con el análisis de la morfometría. La morfometria es una herramienta de diagnóstico de las condiciones fisiográficas naturales y sus parámetros de análisis, tales como: factor de forma, densidad de drenaje, pendiente del terreno, etc., que sirven como indicadores de la susceptibilidad a la degradación del medio ambiente. Es decir, sirve para orientar las acciones de planificación, gestión y mitigación para la conservación y uso de los recursos naturales.

Por lo tanto, esta información debe ser detallada y cuantificada utilizando un Sistema de Información Geográfica (SIG).

Este trabajo tiene como objetivo principal obtener y calcular los parámetros morfométricos de una cuenca con la ayuda de un Sistema de Información Geográfica (SIG), como herramienta para ayudar en la recopilación de datos así como en la toma de decisiones. Como objetivos específicos se plantean:

- Creación de un campo de base de datos de estudio.

- Medición de parámetros morfométricos a través de SIG.

- Cálculo de morfometría y discusión de los resultados.

\section{Materiales y métodos}

\subsection{Material}

\subsubsection{Material Cartográfico}

Los datos de drenaje y de altimetría fueron generados mediante la vectorización de tablas topograficas IGC a escala 1: 10000 con una equidistancia vertical entre contornos de $5 \mathrm{~m}$, utilizando un SIG. Estas tablas están disponibles en Geoportal (IGC, 2014) y ya georreferenciadas en el sistema de coordenadas geográficas WGS 1984. De ellas se extrae información sobre el drenaje y la sostenibilidad de los arroyos que conforman la cuenca, los contornos y sus respectivas cotas y los puntos de mayor altitud. 


\subsection{2 Área de estudio}

La cuenca "C" está totalmente insertada en el Parque Estadual da Serra do Mar (Núcleo CunhaIndaiá, no município de Cunha-SP), situado entre las coordenadas geográficas: 23o14'29" a 23o15'10" latitud (S) y 45o01'10" a 45o01'50" longitud (W) (Fig 1). La cuenca desemboca en el Rio Paraibuna y presentan una variación de altitud de entre $1.030 \mathrm{~m} 1.188 \mathrm{~m}$ y un área total de $0.67 \mathrm{~km}^{2}$.

Según la clasificación Köppen, el clima local es el Cfb, es decir, templado húmedo. La temporada de lluvias se extiende de octubre a marzo y el período seco, o ligeramente húmedo, comprende los meses de abril a septiembre. La temperatura media anual del aire es $16.5^{\circ} \mathrm{C}\left(10\right.$ años), con una media de $10^{\circ} \mathrm{C}$ a $22^{\circ} \mathrm{C}$ en invierno y en verano respectivamente (Silva, et al., 2005). La precipitación media anual es de 2.276 mm (Ranzini, 2002).

\subsubsection{Sistema informático}

Para la generación y el análisis de la base de datos espaciales se utilizó el programa ArcMap 10.1, que se utiliza en la vectorización de la red de drenaje y de las curvas de nivel, la reproyección mapas, delineación y medición de parámetros morfométricos de la cuenca.

\subsection{Métodos}

\subsection{1 Índices Morfométricos}

Para obtener los índices morfométricos, los mapas de altitud han sido proyectados en el sistema de coordenadas planas UTM WGS 1984 zona 23 S, en un SIG.

\subsubsection{Parámetros Dimensionales de la microcuenca}

Se obtuvieron los parámetros dimensionales: mayor longitud de la cuenca (C), que corresponde a la dirección del valle principal, entre los puntos inicial y final de la línea de la cuenca (Schum, 1956); anchura mayor de la cuenca (L), que corta a través del valle principal (Strahler, 1957); longitud del cauce principal (Cp), longitud total de la red de drenaje (Cr), acompañando a la sinuosidad del cauce principal y sus afluentes (Horton, 1945); perímetro de la cuenca (P) correspondiente a la longitud de la línea de la cuenca; y el área de la cuenca (A) circunscrito por la línea de la cuenca que define.(Rodrigues e Pissarra, 2008; Rodrigues, et al., 2013, Rodrigues, 2014).

\subsubsection{Características relacionadas a la Forma}

A partir de los datos dimensionales obtenidos se calcularon las variables relacionadas con la topografía y el drenaje de la cuenca:

Factor de forma (Ff), se obtuvo mediante la Ecuación (1), propuesta por Horton (1945).

$$
F f=\left(\frac{A}{p^{2}}\right)
$$


Donde: $A$ es el área de drenaje en $\mathrm{km}^{2}$ y $p$ es el perímetro de la cuenca en $\mathrm{km}$.

El índice de circularidad (Ic), que permite comparar la forma de la cuenca con la geometría del círculo (Ecuación 2):

$$
\text { Ic }=12.57 \cdot\left(\frac{A}{p^{2}}\right)
$$

Donde: $A$ es el área de drenaje en $\mathrm{km}^{2}$ y $p$ es el perímetro en $\mathrm{km}$.

El coeficiente de compacidad (Kc) se refiere también a la forma de la cuenca con un círculo, de acuerdo a Villela \& Mattos (1975), este coeficiente es un número adimensional que varía con la forma de la cuenca, independientemente de su tamaño. Para su determinación se utilizó la Ecuación (3).

$$
K c=0.28 \cdot\left(\frac{P}{\sqrt{A}}\right)
$$

Donde: $\mathrm{P}$ es el perímetro en $\mathrm{m}$ y $\mathrm{A}$ es el área de drenaje en $\mathrm{m}^{2}$.

Cuando los resultados de factor de forma, el coeficiente compacidad y el índice de circularidad son iguales o cercanos a 1.0, la cuenca se acerca a la forma circular con alta tendencia a inundar. Cuando el coeficiente de compacidad es mayor que 1.7, corresponde a una larga cuenca con mayor tendencia a la conservación del medio ambiente de acuerdo a la Tab. 1 .

Tabla 1. Los valores y la interpretación de los resultados en cuanto a: factor de forma (Ff), el índice de circularidad (Ic) y el coeficiente de compacidad (Kc) de la cuenca.

\begin{tabular}{|c|c|c|c|c|}
\hline Ff & Ic & Kc & Formato dela Cuenca & Interpretación Ambiental \\
\hline $1.00-0.75$ & $1.00-0.8$ & $1.00-1.25$ & Circular & Alta tendencia a inundaciones \\
$0.75-0.50$ & $0.8-0.6$ & $1.25-1.50$ & Ovalada & Mediana tendencia a inundaciones \\
$0.50-0.30$ & $0.6-0.40$ & $1.50-1.70$ & Oblongo & Baja tendencia a la inundación \\
$<0.30$ & $<0.40$ & $>1.70$ & Largo & Cuencas propensas a la conservación \\
\hline
\end{tabular}

Adaptado de fuente: Villela e Matos (1975), citado por Rodrigues (2014).

\subsubsection{Características de Drenaje}

Las variables relacionadas con el drenaje fueron: el orden de la cuenca, la densidad de drenaje, los gradientes de canales, la extensión de la trayectoria de flujo de superficie, razón de textura y el índice de sinuosidad de canales (Rodrigues et al., 2013). El orden de la cuenca (W) es una clasificación de la red de drenaje con la identificación y cuantificación de todos los canales (Strahler, 1957). La densidad de drenaje (Dd), según Horton (1945) corresponde a la relación entre la longitud total de la cuenca del río y su respectiva área, que se expresa en $\mathrm{km}$ de ríos por $\mathrm{km}^{2}$. França (1968) clasificó la densidad de drenaje de acuerdo con la Tab. 2.

El gradiente de canales es la relación entre la altura o dimensión máxima y la longitud del canal principal. Según Horton (1945), el gradiente de canales indica la pendiente de los cursos de agua y se calcula de la siguiente forma: 
Tabla 2. Valores e interpretación de los resultados de la densidad de drenaje

\begin{tabular}{|c|c|c|}
\hline Dd (valores) & Interpretación & La interpretación ambiental de la cuenca \\
\hline$<1.5$ & Bajo & Baja la escorrentía y aumenta la infiltración \\
\hline $1.5-2.5$ & Medio & Tendencia media de escorrentía \\
\hline $2.5-3.0$ & Alto & Alta tendencia a fluir ya las inundaciones \\
\hline$>3.0$ & muy Alta & Alta tendencia a la escorrentía, y a la erosión \\
\hline
\end{tabular}

Fuente: Horton (1945), Strahler (1957) y França (1968), citado por Rodrigues (2014).

$$
G r=\left(\frac{A M}{C c p}\right) \cdot 100
$$

Donde: Gr es el gradiente de canales expresado en \%, AM es la máxima altitud en $\mathrm{m}$ y Ccp es la longitud del canal principal en $\mathrm{m}$.

La extensión de la ruta de la superficie (EPS) de agua de la inundación es la distancia media recorrida por las inundaciones antes de encontrar un canal permanente, expresado en metros. La longitud de la trayectoria de la superficie se calcula según Christofoletti, 1969.

$$
\text { Eps }=\left[\frac{1}{2} \cdot D d\right] \cdot 1000
$$

Donde: Eps es la medida de trayectoria de inundación expresada en m y Dd es la densidad de drenaje en $\mathrm{km} \mathrm{km}^{-2}$

El índice de sinuosidad (Is) del río es una relación entre la longitud real del canal principal y la distancia en línea recta entre dos extremos del canal principal, según la Ecuación (6).

$$
I s=\left(\frac{C c p}{D v}\right)
$$

Donde:Ccp es la longitud del canal en m y Dv es lo vector de distancia entre dos extremos del canal principal en $\mathrm{m}$.

Los valores cercanos a 1indican que el canal tiende a ser recto. Valores superiores a 2 indican canales tortuosos (Lana, 2001).

\subsubsection{Características del relieve de la cuenca}

En la evaluación del relievese obtiene la amplitud altimétrica $(H)$ en metros, que es la diferencia entre la cota más elevada aguas arriba y la cota más baja aguas abajo de la cuenca. La razón de alivio (Rr), según Schumm (1956), es la relación entre la diferencia de altitud de los puntos extremos de la cuenca y su mayor longitud (C), y permite la comparación de la altimetría de las regiones, demostrando que cuanto mayor sea el valor, más desigual es el relieve predominante en la región, lo que indica que la región es más susceptible a la erosión (Rodrigues et al., 2013). La razón del relieve se obtiene según la ecuación (7). 


$$
R c=\frac{H}{C}
$$

Donde $\mathrm{C}$ es la longitud de la cuenca en $\mathrm{m}$.

Para obtener el mapa de pendientes fue necesario generar el modelo de elevación del terreno. En SIG se realizó la interpolación de las curvas de nivel, puntos de elevación y red de drenaje, utilizando celdas de $5 \mathrm{~m}$, a través de la función Topo to Raster de la herramienta Spatial Analyst. Con el resultado de la interpolación se obtuvo la pendiente utilizando la función Slope, contenida en la herramienta 3D Analyst. Las clases de pendiente fueron ordenadas según EMBRAPA (1999) con el intervalo de relieve: 0- $3 \%$, 3-6 \%, 6- $12 \%$, 12- 20\%, 20- 40\% y> 40\%. Estos intervalos se clasifican, respectivamente, como relieve plano, ondulado suave, ondulado, ondulado fuerte, montañosa y escarpada.

La pendiente media es la suma de la longitud de las cotas multiplicada por la equidistancia entre ellas y dividida por el área de la cuenca, se obtiene mediante la Ecuación (8) (Lima, 1986; citado por Rodrigues et al., 2013).

$$
D \%=\frac{(\Sigma C n \cdot \Delta H)}{A} \cdot 100
$$

Donde, D\% es la pendiente media, expresada en \%, Cn es la longitud de las cuotas en $\mathrm{m}$, H es la equidistancia entre las elevaciones en $\mathrm{m}$ y A es el área en $\mathrm{km}$.

El coeficiente de rugosidad (Rn) según Baracuhy et al., (2003), se obtiene multiplicando la densidad por la pendiente media de drenaje de la cuenca y es un parámetro ambiental que dirige el uso potencial del suelo. En la interpretación del coeficiente de rugosidad se consideró la clasificación propuesta por Rocha (1997), donde los coeficientes de rugosidad se dividen en cuatro clases: A: terreno propicio para la agricultura; $\mathrm{B}$ : terreno propicio para el pastoreo; $\mathrm{C}$ : terreno propicio para el pasto y la reforestación y; D: terreno propicio sólo para la reforestación, de acuerdo con la Tab. 3 .

Tabla 3. Valores einterpretación del coeficiente de rugosidad

\begin{tabular}{|c|c|c|}
\hline Coeficiente de rugosidad & Clases & uso del suelo \\
\hline $1.09-10.63$ & A & Agricultura \\
\hline $10.64-20.18$ & B & Pasto \\
\hline $20.19-29.73$ & C & pastos / reforestación \\
\hline$>29.74$ & D & repoblaciónforestal \\
\hline
\end{tabular}

El coeficiente de rugosidad se obtiene según la Ecuación (9).

$$
R n=(D d \cdot D \%)
$$

Donde Rn es el coeficiente de rugosidad, Dd es la densidad de drenaje en $\mathrm{km} \mathrm{km}^{-2}$ y $\mathrm{D} \%$ la pendiente media $\%$. 


\section{Resultados y discusión}

\subsection{Análisis morfométrico}

$\mathrm{El}$ área de la cuenca "C" núcleo Cunha se estimó en $0.67 \mathrm{~km}^{2}$. El perímetro de la cuenca resultó ser de $3.78 \mathrm{~km}$. El resto de factores que se relacionan con los parámetros morfométricos se muestran en la Tab. 4.

Tabla 4. Características físicas de la cuenca " $C$ ".

\begin{tabular}{lcc}
\hline \multicolumn{2}{c}{ Características físicas } & \\
\hline Parámetros dimensionales de la cuenca & unidad & resultados \\
\hline Área (A) & $\mathrm{km}$ & 0.67 \\
Perímetro (P) & $\mathrm{km}$ & 3.78 \\
Ancho (L) & $\mathrm{km}$ & 1.36 \\
Longitud (C) & $\mathrm{km}$ & 1.00 \\
DrenajeLongitud(Cr) & $\mathrm{km}$ & 1.18 \\
Longitud de las curvas de nivel (Cn) & $\mathrm{km}$ & 76.97 \\
Longitud del canal principal (Ccp) & $\mathrm{km}$ & 0.93 \\
Vector de Distancia (Dv) & $\mathrm{km}$ & 0.77 \\
\hline
\end{tabular}

La forma de la cuenca es el resultado de un largo proceso geomorfológico, un parámetro indicativo de las condiciones físicas de la cuenca en relación con su estado de conservación. Los resultados relacionados con el factor de forma, índice de circularidad y la compacidad coeficiente se pueden observar en Tab. 5.

Tabla 5. Características relacionadas con la forma de las cuencas hidrográficas "C".

\begin{tabular}{lcc}
\hline Características relacionadas a la forma de la cuenca & Unidad & Resultados \\
\hline Factor de forma (Ff) & - & 0.66 \\
Índice de circularidad (Ic) & - & 0.60 \\
Coeficiente de compacidad(kC) & - & 1.30 \\
\hline
\end{tabular}

De acuerdo con la clasificación propuesta por Villela y Matos (1975), la cuenca "C" tiene la forma ovalada, mostrando por tanto una tendencia a inundar mediana.

Tabla 6. Patrones de drenaje de la cuenca "C".

\begin{tabular}{lcc}
\hline Los patrones de drenaje de la cuenca & Unidad & Resultados \\
\hline Orden de la cuenca (W) & - & $2^{\mathrm{a}}$ \\
Densidade de drenaje (Dd) & $\mathrm{km} / \mathrm{km}_{-}$ & 1.77 \\
Coeficiente de Mantenimiento (Cm) & $\mathrm{m} / \mathrm{m}_{-}$ & 0.57 \\
Extensión de la inundación superficial (Eps) & $\mathrm{m}$ & 883.47 \\
Gradiente de canales (Gc) & $\%$ & 127.88 \\
Índice de sinuosidad (Is) & - & 1.20 \\
\hline
\end{tabular}


Tabla 7. Orden, número y longitud de los cuerpos de agua en la cuenca hidrográfica "C".

\begin{tabular}{ccc}
\hline Orden $(\mathbf{W})$ & Número & Longitud $(\mathbf{m})$ \\
\hline $1^{\mathrm{a}}$ & 2 & 775.39 \\
$2^{\mathrm{a}}$ & 1 & 403.96 \\
TOTAL & 3 & 1179.35 \\
\hline
\end{tabular}

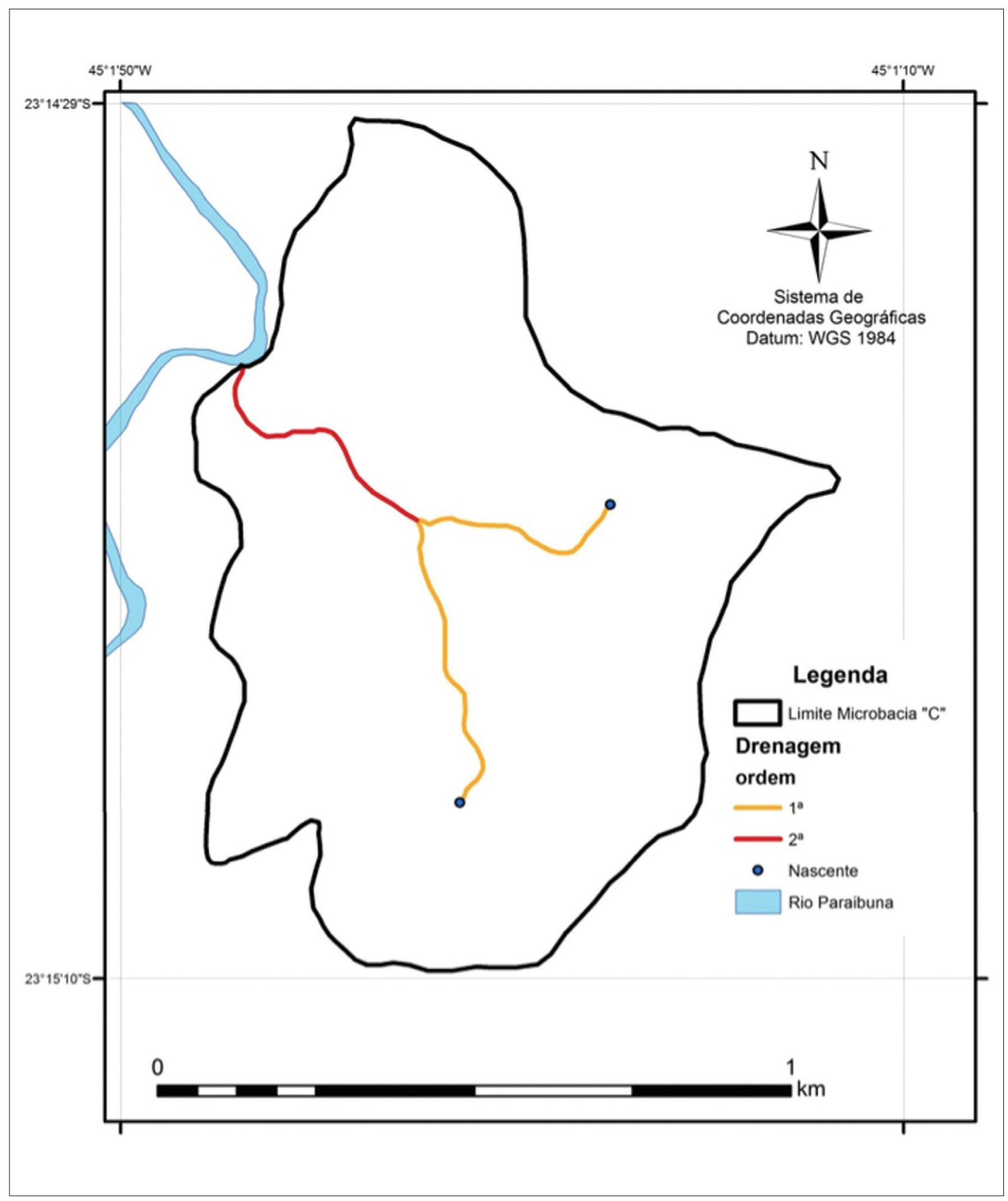

Figura 2. Distribución de los cursos de agua de la cuenca "C". 
La densidad de drenaje estimada fue de $1.77 \mathrm{~km}$ de río por $\mathrm{km}^{2}$ de área, considerada como promedio, según la clasificación de Horton (1945), presentando una tendencia mediana a la escorrentía. Otras medidas relacionadas con los patrones de drenaje se pueden observar en Tab. 6 .

El orden (W), fue identificado y cuantificado para los canales de drenaje y sus respectivas órdenes de conformidad según la Tab. 7. La cuenca se clasificó como de segundo orden, según el sistema de clasificación propuesto por Strahler (1957), La Fig. 2 muestra la disposición de los cuerpos de agua y sus respectivas órdenes.

Las características relacionadas con el alivio de la cuenca se muestran en la Tab. 8. La altitud máxima de la cuenca es $1188 \mathrm{~m}$ y la altitud mínima es $1030 \mathrm{~m}$, lo que resulta en una elevación promedio de 1109 m y una amplitud de altimetría de $158 \mathrm{~m}$. El coeficiente de rugosidad es 101.88, lo que indica que el área de la cuenca pertenece a la clase $\mathrm{D}$, con terreno favorable para la reforestación. La cuenca también tiene una inclinación de aproximadamente un $57.7 \%$, siendo considerado como el relieve acantilado de acuerdo con la clasificación de EMBRAPA (2006).

Tabla 8. Características de las cuencas hidrográficas alivio "C".

\begin{tabular}{lcc}
\hline Características del relieve de la cuenca & Unidad & Resultados \\
\hline pendiente media (D) & $\%$ & 57.66 \\
altitud media (Hm) & $\mathrm{m}$ & 1109 \\
altitud máxima (AM) & $\mathrm{m}$ & 1188 \\
altitud mínima (Am) & $\mathrm{m}$ & 1030 \\
amplitud altimetría de la cuenca (H) & $\mathrm{m}$ & 158 \\
Razón de alivio (Rr) & - & 0.16 \\
Coeficiente de rugosidad (Rn) & - & 101.88 \\
\hline
\end{tabular}

\section{Conclusiones}

La cuenca debe permanecer con la protección del suelo en terreno montañoso con el bosque Atlántico, ya que debido a sus escarpadas laderas (pendientes del $57,7 \%$ ), es susceptible a la escorrentía del agua, la erosión y la sedimentación de los ríos durante una elevada precipitación. El uso de un sistema de información geográfica es esencial para este tipo de estudios.

El elevado coeficiente de rugosidad (101.88) ajusta a la cuenca a la clase D, con terreno favorable para la reforestación.

Los valores del factor de forma, el índice de circularidad y compacidad coeficiente, indican una tendencia mediana a fluir debido a la forma oval de la cuenca. Se puede inferir por tanto la presencia de una baja infiltración en relación a escorrentía y en ausencia de cobertura forestal. 


\section{Referencias}

Baracuhy, J.G.V., Kurtz, S.M.J.M., Kurtz, F.C., Duarte, S.M.A., Lima, V.L.A., Rocha, J.S.M., Neto, J.D., 2003. Deterioração físico-conservacionista da microbacia hidrográfica do riacho Paus Brancos, Campina Grande, PB. Revista Brasileira de Engenharia Agrícola e Ambiental, 7, 159-164.

Carvalho, P.R.S., Guimarães, R.F., Júnior, O.A.C., 2010. Análise comparativa de métodos para delimitação automática das sub-bacias do alto curso do rio preto. Espaço \& Geografia, 13 (2) 227-307.

Cherem, L.F.S., Magalhães, A.P., Faria, S.D., 2011. Análise e compartimentação morfométrica da bacia hidrográfica do alto Rio das Velhas - Região central de Minas Gerais. Revista Brasileira de Geomorfologia, 12, 11-21.

EMBRAPA, 2006. Centro Nacional de Pesquisa de Solos. Sistema brasileiro de classificação de solos. Rio de Janeiro. 256-260.

França, G.V., 1968. Interpretação fotográfica de bacias e redes de drenagem aplicada a solos da região de Piracicaba. Tese de Doutorado. Escola Superior de Agricultura "Luiz de Queiroz", Universidade de São Paulo.

Horton, R.E., 1945. Erosion al development of streams and their drainage basins: hydrographical approach to quantitative morphology. Geological Society of America Bulletin, 56. 275-370.

IGC, 2014. Instituto geográfico e cartográfico, Geoportal. Disponível em: http://geoportal. igc.sp.gov.br:8080/GeoPortalIGC/Internet/. Acesso em 12 de ago. 2014.

Lana, C.L., Alves, J.M.P., Castro, P.T.A., 2001. Análise morfométrica da bacia do rio Tanque, MG-Brasil, Revista da Escola de Minas; 54, 121-126.

Lima, W.P., 1986. Princípios de hidrologia florestal para o manejo de bacias hidrográficas. Tese de Doutorado. Escola Superior de Agricultura “Luiz de Queiroz”, USP. São Paulo.

Ranzini, M., 2002. Modelagem hidrológica de uma microbacia florestada da Serra do Mar, SP, com o modelo TOPMODEL - simulação do comportamento hidrológico em função do corte raso. São Carlos. Tese de Doutorado. Escola de Engenharia de São Carlos, Universidade de São Paulo.

Rocha, J.S.M., 1997. Manual de projetos ambientais. Universidade Federal de Santa Maria, Rio Grande do Sul, Brasil.

Rodrigues, V.A., 2014. Avaliação dos processos hidrológicos em microbacias hidrográficas. Tesis Prof. Adjunto. Universidade Estadual Paulista - Faculdade de Ciências Agronômicas. UNESP. Botucatu. São Paulo. Brasil. 125p.

Rodrigues, V.A., 2004. Morfometria e mata ciliar da microbacia hidrográfica. In: Anais do VIII Workshop em Manejo de Microbacias Hidrográficas. UNESP. Botucatu. 7-18.

Rodrigues, V.A., Cardoso, L.G., Pollo, R.A., Ré, D.S., Pissarra, T.C., Valle Júnior, R.F., 2013. Análise morfométrica da microbacia do Ribeirão das Araras. Revista Científica Eletrônica de Engenharia Florestal, 21, 25-37.

Rodrigues, F.M., Pissarra, T.C.T., Campos, S. 2008. Caracterização morfométrica da microbacia hidrográfica do córrego da fazenda Glória, município de Taquaritinga, SP. Irriga, v. 13, 310-322.

Villela, S.M., Mattos, A., 1975. Hidrologia aplicada. São Paulo, Brasil.

Schumm, S.A., 1956. Evolution of drainage systems and slopes in badlands at Perth Ambory, 
New Jersey. Bulletin of the Geological Society of America, 67, 597-645.

Silva, A.M., 1995. Princípios básicos de hidrologia. Lavras: UFLA, Minas Gerais, Brasil.

Silva, A.M., Ranzini, M., Arcova, F.C.S., Cicco, V., 2005. Estudo integrado do processo erosivo numa microbacia experimental localizada no município de Cunha - SP. São Paulo, UNESP, Geociências, 24, 43-53.

Strahler, A.N., 1957. Quantitative analyses of watershed geomorphology. Transactions of American Geophysical Union 38, 913-920. 